\title{
ON THE ABSOLUTE HARMONIC SUMMABILITY OF A SERIES RELATED TO A FOURIER SERIES
}

\author{
O. P. VARSHNEY
}

1.1. Let $\sum a_{n}$ be a given infinite series with the sequence of partial sums $\left\{s_{n}\right\}$. Let the sequence $\left\{t_{n}\right\}$ be defined by

$$
\begin{aligned}
t_{n}=\frac{(n+1)^{-1} s_{0}+n^{-1} s_{1}+\cdots+1 \cdot s_{n}}{} & \\
P_{n} & \left(P_{n}=1+\frac{1}{2}+\cdots+\frac{1}{n+1}\right) .
\end{aligned}
$$

The series $\sum a_{n}$ is defined to be summable by harmonic means if the sequence $\left\{t_{n}\right\}$ tends to a limit as $n \rightarrow \infty$ [4]. If the series $\sum_{n}\left|t_{n}-t_{n-1}\right|$ is convergent, we say that the series is absolutely harmonic summable. It is known that the method of summability is absolutely regular and implies absolute Cesàro summability of every positive order [2].

1.2. Let $f(t)$ be a periodic function, with period $2 \pi$, and integrable $(L)$ over $(-\pi, \pi)$. We assume without any loss of generality that the Fourier series of $f(t)$ is given by

$$
\sum_{1}^{\infty}\left(a_{n} \cos n t+b_{n} \sin n t\right)=\sum_{1}^{\infty} A_{n}(t)
$$

and that $\int_{-\pi}^{\pi} f(t) d t=0$. We write

$$
\phi(t)=\frac{1}{2}\{f(x+t)+f(x-t)\} .
$$

Mohanty [3] has considered the absolute Riesz summability of the series

$$
\sum_{n=1}^{\infty} A_{n}(t) / \log (n+1)
$$

2. In this paper we establish the following theorem:

THEOREM. If $\phi(t)$ is of bounded variation in $(0, \pi)$ then the series (1.22) is absolutely summable by harmonic means.

We require the following lemmas for the proof of our theorem:

Received by the editors December 10, 1958. 
LEMma 1 [5]. Uniformly for $0<t<\pi$

$$
\left|\sum_{m}^{n} \frac{\sin \nu t}{\nu}\right| \leqq K
$$

where $m$ and $n$ are any positive integers.

LEMma 2 [1]. If $0<t<\pi$, then

$$
\left|\sum_{k=0}^{m} \frac{\cos (k+1) t}{k+1}\right|=O\left(1+\log \frac{1}{t}\right) .
$$

With the help of Lemmas 1 and 2, we may easily deduce

Lemma 3. If $0<t<\pi$, then for all positive integers $m$ and $m^{\prime}$

$$
\sum_{k=m}^{m^{\prime}} \frac{\sin (n-k) t}{k+1}=O\left(1+\log \frac{1}{t}\right) .
$$

Lemma 4. If $P_{n}=1+1 / 2+\cdots+1 /(n+1)$, then

(i)

$$
\begin{aligned}
\text { (i) } & \sum_{k=0}^{[n / 2]-2}\left|\Delta\left(\frac{P_{n}(n+1)-P_{k}(k+1)}{(n-k) \log (n-k+1)}\right)\right|=O(1) ; \\
\text { (ii) } & \quad \sum_{k=[n / 2)}^{n-2}\left|\Delta\left(\frac{P_{k}}{k+1} \cdot \frac{1}{\log (n-k+1)}\right)\right|=O\left(\frac{P_{n}}{n}\right) .
\end{aligned}
$$

For proving (i) we observe that

$$
\begin{aligned}
\sum_{k=0}^{[n / 2]-2} \mid \Delta & \left(\frac{P_{n}(n+1)-P_{k}(k+1)}{(n-k) \log (n-k+1)}\right) \mid \\
\leqq & \sum_{k=0}^{[n / 2]-2} \frac{P_{n}(n+1)-P_{k}(k+1)}{(n-k)(n-k-1) \log (n-k+1)} \\
& +\sum_{k=0}^{[n / 2]-2} \frac{P_{k+1}(k+2)-P_{k}(k+1)}{(n-k) \log (n-k+1)} \\
& +O\left(\sum_{k=0}^{[n / 2]-2} \frac{P_{n}(n+1)-P_{k}(k+1)}{(n-k)^{2} \log ^{2}(n-k+1)}\right) \\
= & O\left(\sum_{k=0}^{[n / 2]-2} \frac{1}{n-k}\right)+O\left[\sum_{k=0}^{[n / 2]-2} \overline{(n-k) \log (n-k+1)}\right] \\
& +O\left[\sum_{k=0}^{[n / 2]-2} \frac{1}{(n-k) \log (n-k+1)}\right] \\
= & O\left(\sum_{k=0}^{[n / 2]-2} \frac{1}{(n-k)}\right)=O(1) .
\end{aligned}
$$


Again

$$
\begin{aligned}
\sum_{k=[n / 2]}^{n-2} \mid \Delta & \left(\frac{P_{k}}{k+1} \cdot \frac{1}{\log (n-k+1)}\right) \mid \\
= & O\left(\sum_{k=[n / 2]}^{n-2} \frac{P_{k}}{k+1} \cdot \frac{1}{(n-k) \log ^{2}(n-k+1)}\right) \\
& \quad+O\left(\sum_{k=\{n / 2]}^{n-2} \frac{P_{k}}{(k+1)^{2} \log (n-k+1)}\right) \\
= & O\left(P_{n} / n\right)\left(\sum_{k=[n / 2]}^{n-2} \frac{1}{(n-k) \log ^{2}(n-k+1)}\right)+O\left(\frac{P_{n}}{n^{2}}\right) \cdot(n) \\
= & O\left(P_{n} / n\right) .
\end{aligned}
$$

This proves the lemma completely.

3. Proof of the theorem. Since

$$
t_{n}=\frac{P_{n} u_{0}+P_{n-1} u_{1}+\cdots+P_{0} u_{n}}{P_{n}}, \quad\left(u_{n}=\frac{A_{n}(t)}{\log (n+1)}\right),
$$

we have

$$
\begin{aligned}
t_{n}-t_{n-1} & =\sum_{\nu=0}^{n-1}\left(\frac{P_{\nu}}{P_{n}}-\frac{P_{\nu-1}}{P_{n-1}}\right) u_{n-\nu} \\
& =\frac{1}{P_{n} P_{n-1}} \sum_{\nu=0}^{n-1}\left(\frac{P_{n}}{\nu+1}-\frac{P_{\nu}}{n+1}\right) u_{n-\nu}
\end{aligned}
$$

For the Fourier series of $f(t)$ at $t=k$,

$$
A_{n}=\frac{2}{\pi} \int_{0}^{\pi} \phi(t) \cos n t d t
$$

so that

$$
t_{n}-t_{n-1}=\frac{2}{\pi} \int_{0}^{\pi} \phi(t)\left(\frac{1}{P_{n} P_{n-1}} \sum_{k=0}^{n-1}\left(\frac{P_{n}}{k+1}-\frac{P_{k}}{n+1}\right) \frac{\cos (n-k) t}{\log (n-k+1)}\right) d t .
$$

Thus in order to prove the theorem, we have to establish that

$$
\sum_{n}\left|\int_{0}^{\pi} \phi(t) g(n, t) d t\right|<\infty,
$$

where

$$
g(n, t)=\frac{1}{P_{n} P_{n-1}} \sum_{k=0}^{n-1}\left(\frac{P_{n}}{k+1}-\frac{P_{k}}{n+1}\right) \frac{\cos (n-k) t}{\log (n-k+1)} .
$$


We observe that

$$
\int_{0}^{\pi} \phi(t) g(n, t) d t=-\int_{0}^{\pi}\left(\int_{0}^{t} g(n, u) d u\right) d \phi(t),
$$

and

$$
\sum_{n}\left|\int_{0}^{\pi}\left(\int_{0}^{t} g(n, u) d u\right) d \phi(t)\right| \leqq \int_{0}^{\pi}|d \phi(t)|\left\{\sum_{n}\left|\int_{0}^{t} g(n, u) d u\right|\right\} .
$$

Since, by hypothesis, $\int_{0}^{\pi}|d \phi(t)|<\infty$, it suffices for our purpose to show that, uniformly for $0<t<\pi$,

$$
\sum_{n}\left|\int_{0}^{t} g(n, u) d u\right|<\infty,
$$

or, what is the same thing,

$$
\sum \equiv \sum_{n}\left|\frac{1}{P_{n} P_{n-1}} \sum_{k=0}^{n-1}\left(\frac{P_{n}}{k+1}-\frac{P_{k}}{n+1}\right) \frac{\sin (n-k) t}{(n-k) \log (n-k+1)}\right|<\infty .
$$

Denoting $\tau=[1 / t]$, we have

$$
\begin{aligned}
\sum \leqq & \sum_{1}^{\tau} \frac{1}{P_{n} P_{n-1}}\left|\sum_{k=0}^{n-1}\left(\frac{P_{n}}{k+1}-\frac{P_{k}}{n+1}\right) \frac{\sin (n-k) t}{(n-k) \log (n-k+1)}\right| \\
& +\sum_{\tau+1}^{\infty} \frac{1}{P_{n} P_{n-1}}\left|\sum_{k=0}^{[n / 2]-1}\left(\frac{P_{n}}{k+1}-\frac{P_{k}}{n+1}\right) \frac{\sin (n-k) t}{(n-k) \log (n-k+1)}\right| \\
& +\sum_{\tau+1}^{\infty} \frac{1}{P_{n} P_{n-1}}\left|\sum_{[n / 2]}^{n-1}\left(\frac{P_{n}}{k+1}-\frac{P_{k}}{n+1}\right) \frac{\sin (n-k) t}{(n-k) \log (n-k+1)}\right| .
\end{aligned}
$$

Now since $|\sin (n-k) t| \leqq(n-k) t$ and $P_{n}(n+1) \geqq P_{k}(k+1)$ for $k \leqq n$, we have

$$
\begin{aligned}
\sum_{1} & =\sum_{1}^{\tau} \frac{1}{P_{n} P_{n-1}}\left|\sum_{k=0}^{n-1}\left(\frac{P_{n}}{k+1}-\frac{P_{k}}{n+1}\right) \frac{\sin (n-k) t}{(n-k) \log (n-k+1)}\right| \\
& \leqq A t \sum_{1}^{\tau} \frac{1}{P_{n} P_{n-1}} \sum_{k=0}^{n-1} P_{n} / k+1 \\
& =\text { At } \sum_{1}^{\tau} P_{n} P_{n-1} / P_{n} P_{n-1} \\
& =O(1) .
\end{aligned}
$$

By Abel's transformation and taking $r$ to be a fixed number $>\pi$, 


$$
\begin{aligned}
\sum_{2}= & \sum_{\tau+1}^{\infty} \frac{1}{P_{n} P_{n-1}}\left|\sum_{k=0}^{[n / 2]-1}\left(\frac{P_{n}}{k+1}-\frac{P_{k}}{n+1}\right) \frac{\sin (n-k) t}{(n-k) \log (n-k+1)}\right| \\
= & \sum_{\tau+1}^{\infty} \frac{1 /(n+1)}{P_{n} P_{n-1}}\left|\sum_{k=0}^{[n / 2]-1} \frac{P_{n}(n+1)-P_{k}(k+1) \cdot \sin (n-k) t}{(n-k) \log (n-k+1)}\right| \\
= & O\left[\sum_{\tau+1}^{\infty} \frac{1 /(n+1)}{P_{n} P_{n-1}}\left(\log \frac{r}{t}\right) \frac{P_{n}(n+1)-P_{[n / 2]-1}\left(\left[\frac{n}{2}\right]\right)}{\left(\left[\frac{n}{2}\right]+1\right) \log \left(\left[\frac{n}{2}\right]+2\right)}\right] \\
& +O\left[\sum_{\tau+1}^{\infty} \frac{1 /(n+1)}{P_{n} P_{n-1}}\left(\log \frac{r}{t}\right)^{[n / 2]-2}\left|\Delta \frac{P_{n}(n+1)-P_{k}(k+1)}{(n-k) \log (n-k+1)}\right|\right. \\
= & O\left(\log \frac{r}{t}\right) \sum_{\tau+1}^{\infty} \frac{1 /(n+1)}{P_{n} P_{n-1}}=O\left(\frac{\log r / t}{P_{\tau}}\right) \\
= & O(1),
\end{aligned}
$$

by using Lemmas 3 and 4 (i).

Since $1 /(n+1)(n-k)=1 /(k+1)(n-k)-1 /(n+1)(k+1)$, we have

$$
\begin{aligned}
\sum_{3}= & \sum_{\tau+1}^{\infty} \frac{1}{P_{n} P_{n-1}}\left|\sum_{k=[n / 2]}^{n-1}\left(\frac{P_{n}}{k+1}-\frac{P_{k}}{n+1}\right) \frac{\sin (n-k) t}{(n-k) \log (n-k+1)}\right| \\
\leqq & \sum_{\tau+1}^{\infty} \frac{1}{P_{n} P_{n-1}}\left|\sum_{k=[n / 2]}^{n-1} \frac{P_{n}-P_{k}}{(k+1)} \cdot \frac{\sin (n-k) t}{(n-k) \log (n-k+1)}\right| \\
& +\sum_{\tau+1}^{\infty} \frac{1 /(n+1)}{P_{n} P_{n-1}}\left|\sum_{k=[n / 2]}^{n-1} \frac{P_{k}}{k+1} \cdot \frac{\sin (n-k) t}{\log (n-k+1)}\right| \\
= & \sum_{31}+\sum_{32} \text {, say. }
\end{aligned}
$$

Now since for $k \geqq[n / 2], P_{n}-P_{k}=O(1)$, we obtain

$$
\begin{aligned}
\sum_{31} & =\sum_{\tau+1}^{\infty} \frac{1}{P_{n} P_{n-1}}\left|\sum_{k=[n / 2]}^{n-1} \frac{\left(P_{n}-P_{k}\right)}{k+1} \cdot \frac{\sin (n-k) t}{(n-k) \log (n-k+1)}\right| \\
& =O\left[\sum_{\tau+1}^{\infty} \frac{1}{P_{n} P_{n-1}}\left|\sum_{k=[n / 2]}^{n-1} \frac{P_{n}-P_{k}}{(k+1)} \cdot \frac{1}{(n-k) \log (n-k+1)}\right|\right] \\
& =O\left(\sum_{\tau+1}^{\infty} \frac{1}{n P_{n} P_{n-1}} \sum_{k=[n / 2]}^{n-1} \frac{1}{(n-k) \log (n-k+1)}\right) \\
& =O\left(\sum_{\tau+1}^{\infty} \frac{\log \log n}{n \log ^{2} n}\right)=O(1) .
\end{aligned}
$$


Finally since $\sum_{a}^{b} \sin n t=O(1 / t)$ for all $a$ and $b$, we have by Abel's transformation

$$
\begin{aligned}
\sum_{32}= & \sum_{\tau+1}^{\infty} \frac{1 /(n+1)}{P_{n} P_{n-1}}\left|\sum_{k=[n / 2]}^{n-1} \frac{P_{k}}{k+1} \cdot \frac{\sin (n-k) t}{\log (n-k+1)}\right| \\
= & O\left(\sum_{\tau+1}^{\infty} \frac{1 /(n+1)}{P_{n} P_{n-1}} \sum_{k=[n / 2]}^{n-2} \frac{1}{t}\left|\Delta\left(\frac{P_{k}}{k+1} \cdot \frac{1}{\log (n-k+1)}\right)\right|\right) \\
& +O\left(\frac{1}{t}\right)\left(\sum_{\tau+1}^{\infty} \frac{1 /(n+1)}{P_{n} P_{n-1}} \cdot \frac{P_{n-1}}{n}\right) \\
= & O\left(\frac{1}{t}\right)\left(\sum_{\tau+1}^{\infty} \frac{1}{n^{2} \log n}\right) \\
= & O\left(\frac{1}{t}\right)\left(\frac{1}{\tau \log \tau}\right) \\
= & O(1),
\end{aligned}
$$

using Lemma 4 (ii).

Collecting (3.1), (3.2), (3.3), and (3.4), we see that the theorem is completely proved.

I am very much indebted to Professor Misra for his kind interest and advice in the preparation of this paper.

\section{REFERENCES}

1. G. H. Hardy and W. W. Rogosinski, Notes on Fourier series, IV. Summability $\left(R_{2}\right)$, Proc. Cambridge Philos. Soc. vol. 43 (1947) pp. 10-25.

2. Leonard McFadden, Absolute Nörlund summability, Duke Math. J. vol. 9 (1942) pp. 168-207.

3. R. Mohanty, On the absolute Riesz summability of a Fourier series and allied series, Proc. London Math. Soc. ser. 2 vol. 52 (1951) pp. 295-320.

4. M. Riesz, Sur l'equivalence de certaines méthodes de sommation, Proc. London Math. Soc. vol. 22 (1924) pp. 412-419.

5. E. C. Titchmarsh, The theory of functions, Oxford, 1939, p. 440.

University of Saugar, Sagar, India 